\title{
Development of a simple model for studying the effects of antifungal agents on multicellular communities of Aspergillus fumigatus
}

Correspondence
Gordon Ramage
g.ramage@dental.gla.ac.uk

Received 21 February 2007

Accepted 14 May 2007

\author{
Eilidh Mowat, ${ }^{1}$ John Butcher, ${ }^{1}$ Sue Lang, ${ }^{1}$ Craig Williams ${ }^{2}$ \\ and Gordon Ramage ${ }^{3}$
}

\author{
${ }^{1}$ Department of Biological and Biomedical Sciences, Glasgow Caledonian University, Glasgow, \\ UK \\ ${ }^{2}$ Microbiology Department, Yorkhill Hospital, Glasgow, UK \\ ${ }^{3}$ Section of Infection and Immunity, Glasgow University Dental School and Hospital, Glasgow, UK
}

\begin{abstract}
Aspergillus fumigatus is an increasingly prevalent opportunistic fungal pathogen of various immunocompromised individuals. It has the ability to form filaments within the lungs, producing dense intertwined mycelial balls, which are difficult to treat. The aim of this study was to develop a suitable model of $A$. fumigatus to examine the effects of antifungal challenge on these intertwined filamentous communities. A. fumigatus NCPF 7367 growth conditions were optimized on both Thermanox coverslips and on flat-bottomed microtitre plates to establish optimal conidial seeding densities. Isolates were treated with itraconazole, voriconazole, amphotericin B and caspofungin and their overall killing efficiency was measured using an XTT formazan metabolic dye assay. This was compared with the CLSI (formerly NCCLS) methodology of broth microdilution of

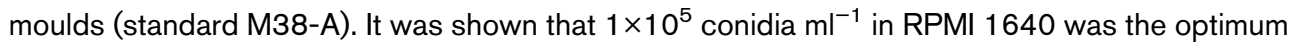
concentration of spores for biofilm formation. Filamentous growth characteristics were not observed until $10 \mathrm{~h}$ incubation, followed by an exponential increase in the biofilm biomass (hyphae and extracellular material) and cellular activity (metabolism). When susceptibility testing of biofilms was compared with that of planktonic cells by CLSI broth microdilution testing, all antifungal drugs were at least 1000 times less effective at reducing the overall metabolic activity of $90 \%$ of the cells. Overall, this study showed that $A$. fumigatus has the ability to form coherent multicellular biofilm structures that are resistant to the effects of antifungal drugs.
\end{abstract}

\section{INTRODUCTION}

Aspergillus species are opportunistic filament-forming moulds. The genus comprises over 180 species, with Aspergillus fumigatus causing the majority of human aspergillus infections (Hope et al., 2005). A. fumigatus is a ubiquitous saprophytic fungus with a worldwide distribution due to the production of small spores called conidia, which have a mean size of $2-3.5 \mu \mathrm{m}$, resulting in the conidia being dispersed into the air and remaining in the atmosphere for time periods that depend on the conditions of the external environment, such as temperature, humidity and seasonal variations (Rivera et al., 2006).

In recent times, the frequency of disseminated fungal infections has increased dramatically. Overall, A. fumigatus

Abbreviations: ABPA allergic bronchopulmonary aspergillosis; AmpB, amphotericin B; CF, cystic fibrosis; CLSM, confocal laser scanning microscopy; IA, invasive aspergillosis; PMIC, planktonic cell MIC; SMIC, sessile cell MIC; XTT, 2,3-bis(2-methoxy-4-nitro-5-sulfo-phenyl)-2Htetrazolium-5-carboxanilide. is now the second most common cause of fungal infection found in hospitalized patients, after Candida albicans (Ellis et al., 2000). Aspergillus conidia are usually eliminated efficiently by the innate and acquired immune systems. However, in immunocompromised patients, such as transplant, leukaemia and human immunodeficiency virus (HIV)-positive patients, A. fumigatus can cause a range of systemic diseases with mortality rates ranging from 30 to $90 \%$ (Brakhage, 2005; Denning et al., 1998; Herbrecht et al., 2002). Pulmonary infection may also occur in other patients such as those with cystic fibrosis (CF). In these patients, infection with $A$. fumigatus may cause allergic bronchopulmonary aspergillosis (ABPA), a mycetoma (fungus ball) or invasive aspergillosis (IA) (de Almeida et al., 2006; Shibuya et al., 2004).

The initial establishment of chronic A. fumigatus infection involves the germination of conidia and subsequent hyphal invasion of the lung tissue (Filler \& Sheppard, 2006). Histological and microscopic examination of bronchopulmonary lavage samples has revealed the presence of 
numerous A. fumigatus hyphae in the form of a complex multicellular structure (mycetoma), which is similar to the biofilms formed by Candida species (Ramage et al., 2001c, 2005). In contrast to the biofilms formed by Candida species, very limited information is currently available on the development and behaviour of A. fumigatus adherent multicellular communities and their response to antifungal treatment. To date, there are only two reports suggesting that Aspergillus species are able to grow and form biofilms (Beauvais et al., 2007; Villena \& Gutierrez-Correa, 2006).

The purpose of this study was to investigate the growth characteristics of filamentous A. fumigatus multicellular communities through the development of an in vitro model that could be utilized to screen the growth characteristics of clinical isolates or mutants, and to examine the antifungal susceptibility profiles of complex biofilm-like structures (Ramage et al., 2002a, c).

\section{METHODS}

Organisms. A. fumigatus NCPF 7367 and four clinical isolates (YHCF1, YHCF2, YHCF3 and YHCF4) obtained from the Royal Hospital for Sick Children (Yorkhill Division, NHS Glasgow, UK) were used throughout this study. All isolates were stored on Sabouraud dextrose agar slopes (Oxoid) at $4{ }^{\circ} \mathrm{C}$.

Growth conditions and standardization of conidial inoculum. $A$. fumigatus was grown on Sabouraud dextrose agar at $37{ }^{\circ} \mathrm{C}$ for $72 \mathrm{~h}$. Conidia were harvested by flooding the surface of the agar plates with $5 \mathrm{ml}$ PBS (Oxoid) containing $0.025 \%(\mathrm{v} / \mathrm{v})$ Tween 20 and rocking gently. The conidial suspension was recovered and dispensed into a $5 \mathrm{ml}$ sterile glass bottle. The conidia were counted using a Neubauer haemocytometer and adjusted to the required concentration in RPMI 1640 (Sigma) buffered to $\mathrm{pH} 7.0$ with 0.165 M MOPS. All procedures were carried out in a HERASafe laminate flow cabinet (model K515; Kendro).

Biofilm formation. A. fumigatus biofilms were formed on commercially available, pre-sterilized, polystyrene, flat-bottomed, 96-well microtitre plates (Corning). Biofilms were formed by adding $200 \mu \mathrm{l}$ of a standardized cell suspension in MOPS-buffered RPMI 1640 to each well for selected time periods $(4,8,12,24$ and $48 \mathrm{~h})$, and incubating statically at $37{ }^{\circ} \mathrm{C}$. A minimum of 12 replicates was performed for each experimental parameter, plus suitable controls. At each selected time point, the medium was aspirated and the biofilms were washed thoroughly three times with sterile PBS by repeated pipetting to remove non-adherent cells.

Confocal laser scanning microscopy (CLSM). A. fumigatus biofilms were formed as described above on the surface of $13-\mathrm{mm}$ diameter sterile Thermanox plastic cell culture coverslips (Nunc) in 24-well tissue culture plates (Nunc). After incubation at $37^{\circ} \mathrm{C}$ for various time periods $(0,2,4,8,10,12,16,18$ and $24 \mathrm{~h})$, the coverslips were washed in sterile PBS and stained using the LIVE/DEAD fluorescent stain (Molecular Probes), according to the manufacturer's instructions. The FUN1 component of the kit was used, which is a bright green fluorescent intracellular stain. This was applied to the washed biofilms for $20 \mathrm{~min}$ in the dark. The biofilms were then washed in PBS and mounted on a slide. The fluorescent filamentous biomass was examined using a Zeiss Axiovert LSM510 confocal microscope attached to an LSM510 laser scanning system with a 488 argon ion laser at $\times 200$ magnification. Sections of the $x y$ plane were taken at $1 \mu \mathrm{m}$ intervals along the $z$-axis to determine the depth of the biofilms and overall physical ultrastructure. Three-dimensional images were obtained using computer software.

Biofilm quantification. Biofilm biomass was assessed using a modified version of a protocol first developed by Christensen et al. (1985) and subsequently modified by O'Toole \& Kolter (1998). At each time interval, the spent culture medium was removed from each well and the adherent cells were washed three times with PBS. These were air-dried and $100 \mu \mathrm{l}$ of $0.5 \%$ (w/v) crystal violet solution was added for $5 \mathrm{~min}$. The solution was then removed by carefully rinsing the biofilms under running water until excess stain was removed. The biofilms were destained by adding $100 \mu 195 \%$ ethanol to each well. The ethanol was gently pipetted to completely solubilize the crystal violet for $1 \mathrm{~min}$, the ethanol was transferred to a clean 96-well microtitre plate and the $A_{570}$ was read (FLUO Star Optima fluorescence microplate reader; BMG Labtech). The absorbance values are proportional to the quantity of biofilm biomass, which comprises hyphae and extracellular polymeric material (the greater the quantity of biological material, the higher the level of staining and absorbance).

2,3-bis(2-Methoxy-4-nitro-5-sulfo-phenyl)-2H-tetrazolium-5carboxanilide (XTT) reduction assay. A semi-quantitative measure of each biofilm was calculated using an XTT reduction assay, adapted from previous studies (Hawser et al., 1998). This is a metabolic reduction assay that measures the activity of cells and can be used to compare untreated cells with cells treated with antimicrobial agents, and has been used previously in studies to evaluate the antifungal sensitivities of filamentous fungi (Antachopoulos et al., 2006; Meletiadis et al., 2001b, c). Briefly, XTT (Sigma) was prepared in a saturated solution at $0.5 \mathrm{~g} \mathrm{l}^{-1}$ in PBS. The solution was filtersterilized through a $22 \mu \mathrm{m}$ pore size filter, aliquoted and stored at $-80{ }^{\circ} \mathrm{C}$. Prior to each assay, an aliquot of stock XTT was thawed and menadione ( $10 \mathrm{mM}$ prepared in acetone; Sigma) was added to a final concentration of $10 \mu \mathrm{M}$. A $100 \mu \mathrm{l}$ aliquot of XTT/menadione solution was added to each well and to appropriate control wells to measure background XTT reduction levels. The plates were incubated in the dark for $3 \mathrm{~h}$ at $37{ }^{\circ} \mathrm{C}$ and the colour change was measured using a $490 \mathrm{~nm}$ filter in a microplate reader (FLUO Star Optima; BMG Labtech). The colorimetric change in the XTT reduction assay directly correlates with the metabolic activity of the biofilm.

\section{End-point susceptibility testing}

M38-A broth microdilution testing. Planktonic cell MICs (PMICs) were evaluated using the CLSI (formerly NCCLS) M38-A standard methodology (NCCLS, 2002). The antifungal agents itraconazole (Sigma), voriconazole (Pfizer), amphotericin B (AmpB; Bristol Myers Squibb) and caspofungin (Merck) were prepared as stock solutions in DMSO (Sigma) and diluted in MOPS-buffered RPMI 1640 to working concentrations. Microtitre plates containing $100 \mu \mathrm{l}$ of each antifungal were serially diluted twofold to produce a concentration range of $0.03-16 \mathrm{mg} \mathrm{l}^{-1}$. A range of conidial suspensions was then prepared in MOPS-buffered RPMI 1640 to a final concentration of $0.4-5 \times 10^{4}$ conidia $\mathrm{ml}^{-1}$ and $100 \mu \mathrm{l}$ was added to each well. The plates were incubated for $48 \mathrm{~h}$ at $35{ }^{\circ} \mathrm{C}$. The PMIC for each antifungal was defined as the lowest concentration that produced complete visible inhibition of growth. Testing of these isolates was performed in quadruplicate.

Modified M38-A broth microdilution testing. For antifungal susceptibility testing of biofilms [sessile cell MICs (SMICs)], conidia were prepared as described above and standardized to a density of $1 \times 10^{6}$ conidia $\mathrm{ml}^{-1}$ in MOPS-buffered RPMI 1640 . Biofilms were formed by pipetting standardized conidial suspensions into selected wells of a microtitre plate and incubating for $24 \mathrm{~h}$ at $35^{\circ} \mathrm{C}$, as 
described above. After growth, the medium was aspirated and nonadherent cells were removed by thorough washing of the cells (three times) using sterile PBS and gentle pipetting. Residual PBS from each well was removed by blotting with paper towels. All antifungals (itraconazole, voriconazole, AmpB and caspofungin) were prepared as described above to provide a working concentration of $512 \mathrm{mg} \mathrm{l}^{-1}$ in MOPS-buffered RPMI 1640. These were serially diluted twofold (1-256 $\mathrm{mg} \mathrm{l}^{-1}$ ) directly into adjacent wells and the challenged cells were incubated statically for a further $48 \mathrm{~h}$ at $35^{\circ} \mathrm{C}$. A number of antifungal-free wells and biofilm-free wells were also included to serve as positive and negative controls, respectively. SMICs were determined as 50 and $90 \%$ reduction in metabolism compared with the untreated control using the XTT reduction assay described above. Testing of these isolates was performed in quadruplicate.

Statistical analysis. The absorbance values of individual biofilms were compared by one-way analysis of variance and using the Bartlett's test for homogeneity of variances and the Bonferroni's multiple comparison post-test. A value of $P<0.05$ was considered to be significant. Analyses were performed using SPSS 13.0 for Windows.

\section{RESULTS AND DISCUSSION}

A. fumigatus is now a leading fungal pathogen and one of the most significant opportunistic fungi in bone-marrowtransplant patients (Singh, 2005). A. fumigatus is also found in a range of other patient groups, including $\mathrm{CF}$ patients, HIV-positive patients and other immunocompromised individuals (Cimon et al., 2001). The presence of mycetomas in the upper airways, the pulmonary epithelial cells of the alveoli or in the maxillary sinuses provides compelling evidence that $A$. fumigatus biofilms are more prolific and problematic than once thought (Filler \& Sheppard, 2006; Mensi et al., 2004). These infections are typified by intricate networks of hyphae that develop from inhaled conidia (Shibuya et al., 2004). Morphologically, these structures resemble other fungal biofilms, such as those of C. albicans (Ramage et al., 2001c), which are clinically important due to their role in pathogenesis and resistance to antifungal agents (Ramage et al., 2002c, 2005). Therefore, we hypothesized that similar pathogenic traits might be exhibited by this fungus, meriting further investigation.

\section{Standardizing an in vitro model}

To date, there have been no published accounts establishing that filamentous A. fumigatus grows as a biofilm. Histopathological evidence has indicated the presence of mycelial plugs and proliferation of hyphae with acute-angle dichotomous branching (Shibuya et al., 2004), which are complicit with the definition of a biofilm. There are many forms of aspergillosis, ranging from ABPA to IA, and despite different clinical presentations, morphological characteristics remain relatively similar and are typified by intricate mycelial networks. Formation of $A$. fumigatus mycelial aggregates that exhibit classic biofilm characteristics is reported here, i.e. an adherent microbial population, adherent to each other and/or surfaces or interfaces (Costerton et al., 1995). To our knowledge, this study is the first to examine submerged cultures of A. fumigatus in vitro in this growth modality.

A key factor that we noticed early in our studies was the critical importance of conidial seeding density. Clearly, the structural morphology and integrity of these multicellular structures was dependent on the concentration of conidia $(\mathrm{ml} \text { medium })^{-1}$ (Figs 1 and 2), a phenomenon previously identified with $C$. albicans biofilm development (Ramage et al., 2001c). To produce adherent aggregated multicellular populations (biofilms) with a similar morphology to those seen during in vivo aspergillosis lung infection, we developed a model, initially examining conidial seeding density of A. fumigatus NCPF 7367 and four clinical isolates. First, we examined a serial dilution of conidial densities, ranging from 10 to $1 \times 10^{6}$ conidia $\mathrm{ml}^{-1}$, and examined the resultant multicellular structures after a $24 \mathrm{~h}$ inoculation using semi-quantitative metabolic and biomass assays. Fig. 1 illustrates that both the metabolic activity and the biomass of the biofilms exhibited a positive correlation with the conidial seeding density, although at the highest concentrations of conidia the biomass appeared to decline. We subsequently used CLSM, a non-invasive technique that enabled three-dimensional structural imaging and depth measurements of intact multicellular structures on
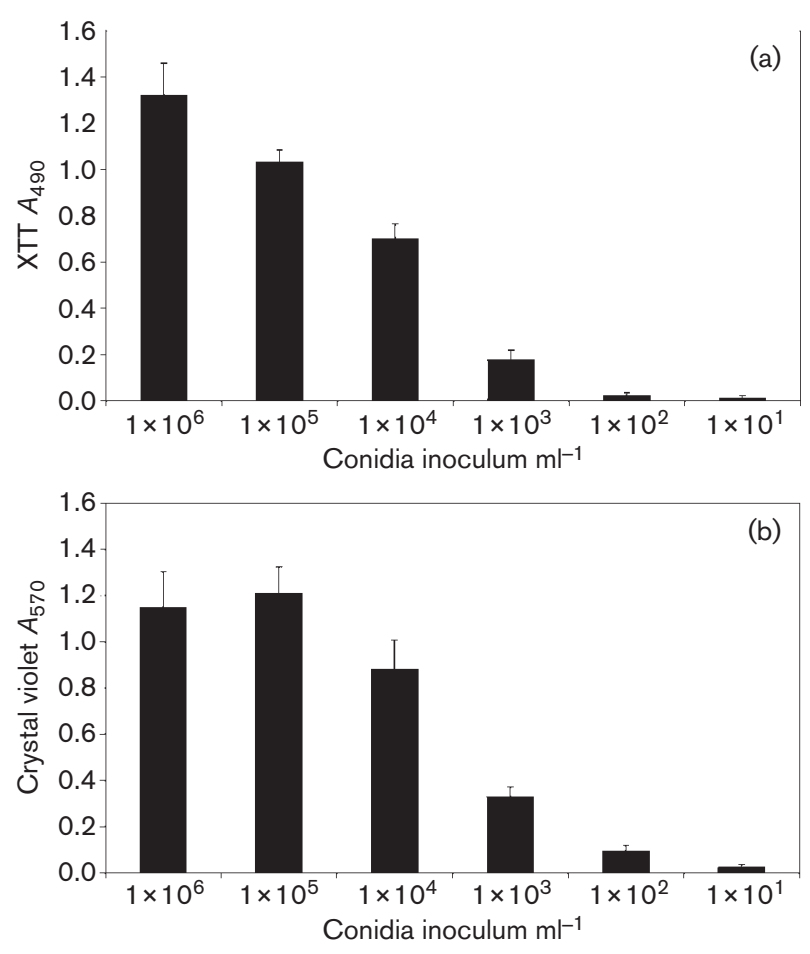

Fig. 1. Effect of conidial concentration on A. fumigatus NCPF 7367 biofilm development after $24 \mathrm{~h}$. Metabolic activity of the biofilm (a) and the biomass (b) increased with the concentration of conidia seeded. Note how there is a limit to the development of the biofilm, as indicated from the biomass generated from an inoculum of $1 \times 10^{6}$ conidia $\mathrm{ml}^{-1}$. 
(a)

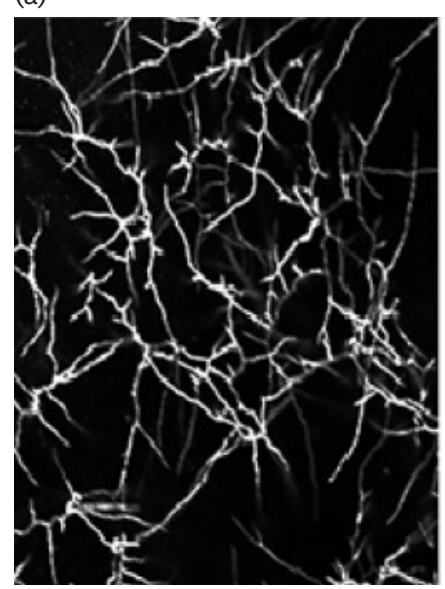

$1 \times 10^{4}$ conidia $\mathrm{ml}^{-1}$

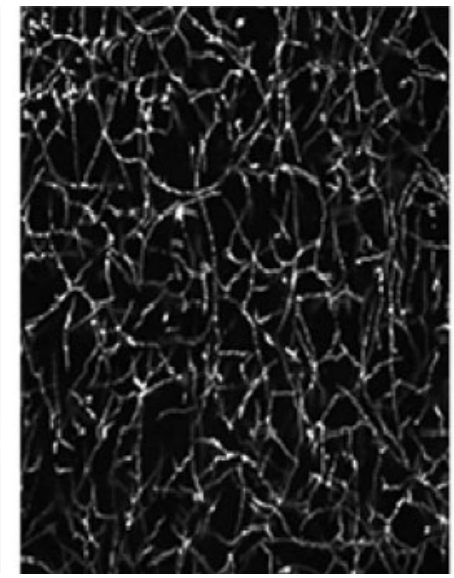

$1 \times 10^{5}$ conidia $\mathrm{ml}^{-1}$

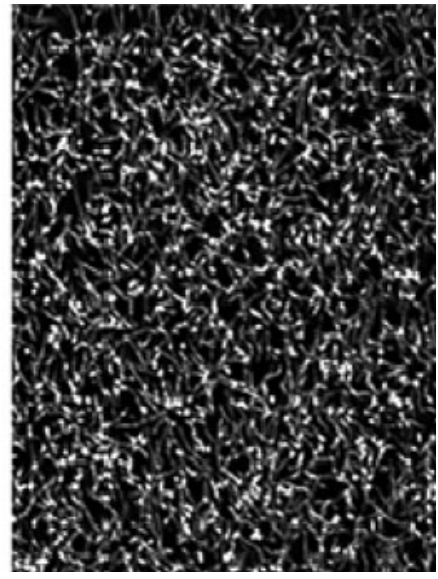

$1 \times 10^{6}$ conidia $\mathrm{ml}^{-1}$

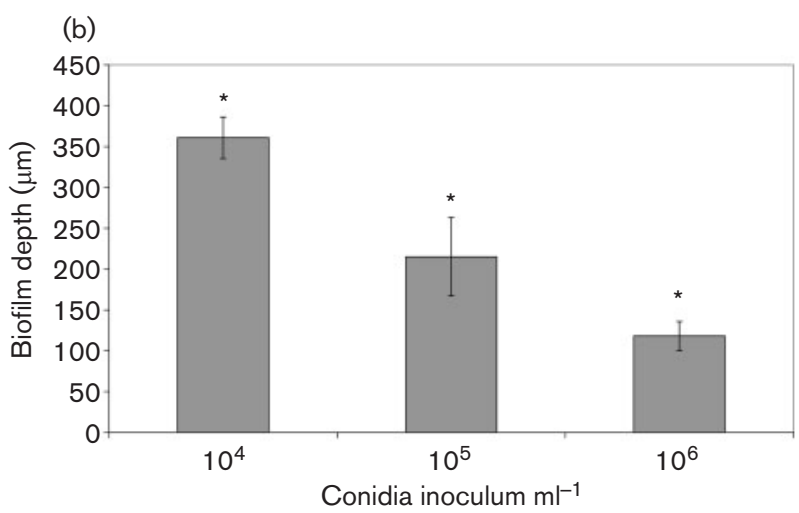

Fig. 2 CLSM of $A$. fumigatus NCPF 7367 biofilms generated using different conidial concentrations. ( $a, b)$ CLSM of biofilms formed by various conidial seeding concentrations on Thermanox coverslips. Differences in structural integrity associated with different conidial density are shown in (a). Higher conidial inoculum concentrations were associated with poorer filamentation and biofilm structural integrity. An inverse relationship was found between conidial density and biofilm depth (b). Significant differences were found between the biofilm depths $\left({ }^{*}, P<0.05\right)$.
Thermanox coverslips. The mature biofilms formed using a series of standardized conidial suspensions $\left(1 \times 10^{4}, 1 \times 10^{5}\right.$ and $1 \times 10^{6}$ conidia $\mathrm{ml}^{-1}$ ) were evaluated by microscopy to investigate the optimal conidial inoculum concentration. Fig. 2(a) illustrates complex multicellular aggregates formed from three different conidial seeding densities after growth for $24 \mathrm{~h}$, which ranged in depth from 117 to $360 \mu \mathrm{m}$. All of the filamentous multicellular structures exhibited acute-angle dichotomous branching to a varying extent. However, the conidial seeding density played an important role in the overall structural integrity of the biofilm structure. Biofilm stability was assessed by shear mechanical force by serially pipetting the biofilms with PBS during the washing procedure (results not shown). Either increasing or decreasing the conidial seeding density 10 fold led to significant differences in the depth of the resultant biofilms $(P>0.05)$. At the highest conidial concentration $\left(1 \times 10^{6}\right.$ conidia $\left.\mathrm{ml}^{-1}\right)$, the biofilms were relatively thin $(117 \mu \mathrm{m})$ and development of filamentous growth was severely restricted, resulting in less overall biomass (Fig. 2). Conversely, biofilms formed by fewer seeded conidia $\left(1 \times 10^{4}\right.$ conidia ml $\left.{ }^{-1}\right)$ were observed to be thicker $(360 \mu \mathrm{m})$ and have longer mycelial frameworks. These were easily disrupted and removed by mechanical forces and were therefore not reproducible. The conidial concentration of $1 \times 10^{5}$ conidia $\mathrm{ml}^{-1}$ produced robust filamentous structures that were resistant to mechanical disruption (Fig. 2). Therefore, this concentration of conidia was selected, as the resultant biofilms exhibited reproducible characteristics that were amenable to the high-throughput testing required for screening of clinical isolates and defined mutants, or for testing the susceptibility of antifungal agents.

\section{Kinetics of biofilm development}

The growth and development of A. fumigatus in the lung, from initial inhalation of conidia to filamentous forms associated with mycetomas, is a key factor in pathogenesis. Clearance of inhaled conidia via innate immune mechanisms in immunocompetent individuals is the key defence against aspergillosis (Latge, 2001). The progression of disease in susceptible patient groups is dependent on the ability of the conidia to germinate and form mycelial masses (mycetomas), which then penetrate the pulmonary epithelium prior to angioinvasion and systemic spread (Filler \& Sheppard, 2006; Kamai et al., 2006). In our studies, we examined the kinetics of multicellular development. Initial adherence, conidial germination, and filamentous growth and differentiation of A. fumigatus 


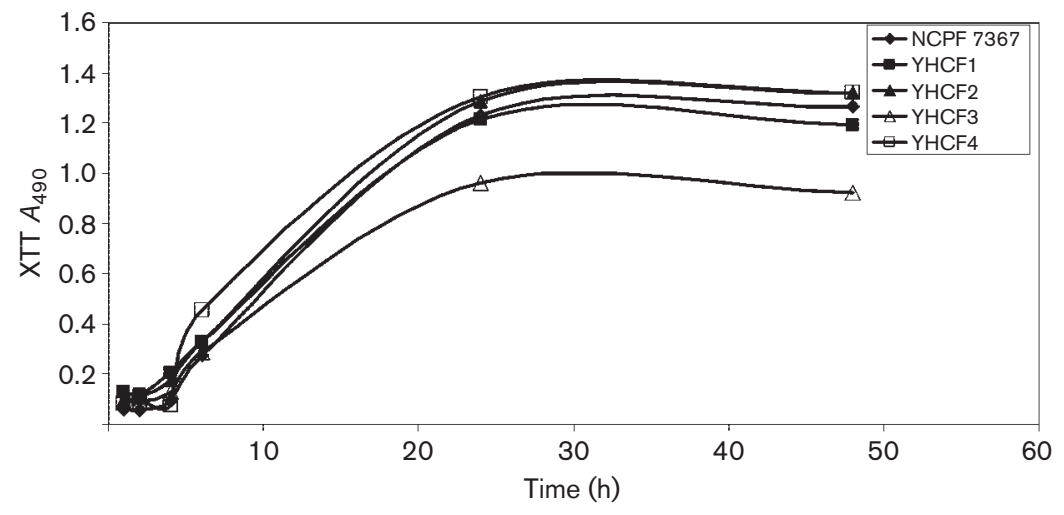

Fig. 3. Growth kinetics of $A$. fumigatus NCPF 7367 and four CF isolates over a $48 \mathrm{~h}$ period (seeding density $1 \times 10^{5}$ conidia $\mathrm{ml}^{-1}$ ).
NCPF 7367 and four clinical isolates were analysed on the surface of polystyrene wells over $48 \mathrm{~h}\left(1 \times 10^{5}\right.$ conidia $\mathrm{ml}^{-1}$ ), as assessed by metabolic activity (XTT) and biomass (crystal violet) measurements (Fig. 3). A correlation between metabolism, biomass and hyphal development was demonstrated. The metabolic activity and the biomass of the biofilms were shown to increase over time. Microscopic analysis revealed that A. fumigatus did not form hyphae until approximately $8 \mathrm{~h}$, in agreement with the results of Meletiadis et al. (2001a), who demonstrated germination at approximately $6 \mathrm{~h}$ using a similar microtitre plate method while examining filamentous growth characteristics of A. fumigatus. When the kinetics of biofilm formation was measured, their results were comparable to those in this study, with a lag phase of approximately $10 \mathrm{~h}$. Hyphae began to intertwine forming a monolayer $(10-16 \mathrm{~h})$, followed by increased structural complexity over the subsequent $4-8 \mathrm{~h}$ (Fig. $4 \mathrm{a}$ ). This was shown more clearly from the depth measurements taken over a $24 \mathrm{~h}$ period. Fig. 4(b) showed an exponential rise in the depth of the biofilm between 10 and $18 \mathrm{~h}$, which then reached a plateau as development ceased and a steady state occurred. These biofilm development characteristics were observed for all isolates tested in this study. The development of the biofilm was slower than that of $C$. albicans biofilms; nevertheless, the overall characteristics of development were similar (Chandra et al., 2001; Ramage et al., 2001c). The slower initial development of mycelia may be related to the overall density of the cells, indicating a potential role for quorum sensing in multicellular $A$. fumigatus populations. This phenomenon has been demonstrated in $C$. albicans with the secreted molecule farnesol, which prevents hyphal development and subsequent biofilm formation (Ramage et al., 2002b).

\section{Antifungal susceptibility testing}

Currently, antifungal therapy remains the main way of controlling the progression of aspergillosis. It is clear from our results that the activity of antifungal agents in vitro is considerably different when biofilms are compared with planktonic cells, and that a number of currently prescribed antifungal agents may be ineffective in the treatment of established biofilm-associated infections. The methodologies used to examine PMICs and SMICs are quite disparate, i.e. there was a $3 \log$ difference in the number of conidia used in the initial inoculum between these assays. This factor may account, in part, for the disparity between the PMICs and SMICs reported here. For example, itraconazole and caspofungin were ineffective against multicellular structures, exhibiting over 1000-fold more resistance than their planktonic counterparts (Table 1).

The current gold standard for choosing the most appropriate treatment for aspergillosis infections is the CLSI standard M38-A broth microdilution in vitro antifungal susceptibility testing assay (NCCLS, 2002). This assay measures inhibition of growth and provides an accurate method for the prediction of fungistatic antifungal drugs. However, in this study we were unable to quantify the effects of the antifungal drugs using this criterion on multicellular structures, which are no longer actively dividing yet are representative of an established infection. ABPA infections with a characteristic mycetoma are typified by greater cellular burdens than are used in planktonic assays and exhibit biofilm characteristics. We used a metabolic dye that exhibited a direct correlation with cellular viability. Viable cell counting was not used due to the inability to correlate colony counts with individual cells. The assay that we described here provides an accurate means of predicting in vivo activity due to the reduced metabolic activity of the cells tested. XTT has been used in other mycological studies to monitor antifungal exposure (Antachopoulos et al., 2006; Hawser et al., 2001; Ramage et al., 2001a). For example, Hawser et al. (2001) previously described an XTT-based methodology for measuring the minimum effective concentrations of antifungal agents against A. fumigatus. In addition, Antachopoulos et al. (2006) recently demonstrated how this simple technology could be used to assess antifungal activity against zygomycetes, and demonstrated a reproducible correlation between XTT-deduced MICs and the CLSI methodology. Ramage and colleagues also showed its utility against both fungal (C. albicans) and bacterial (Pseudomonas aeruginosa) cells, planktonic and sessile cells, and with 
(a)
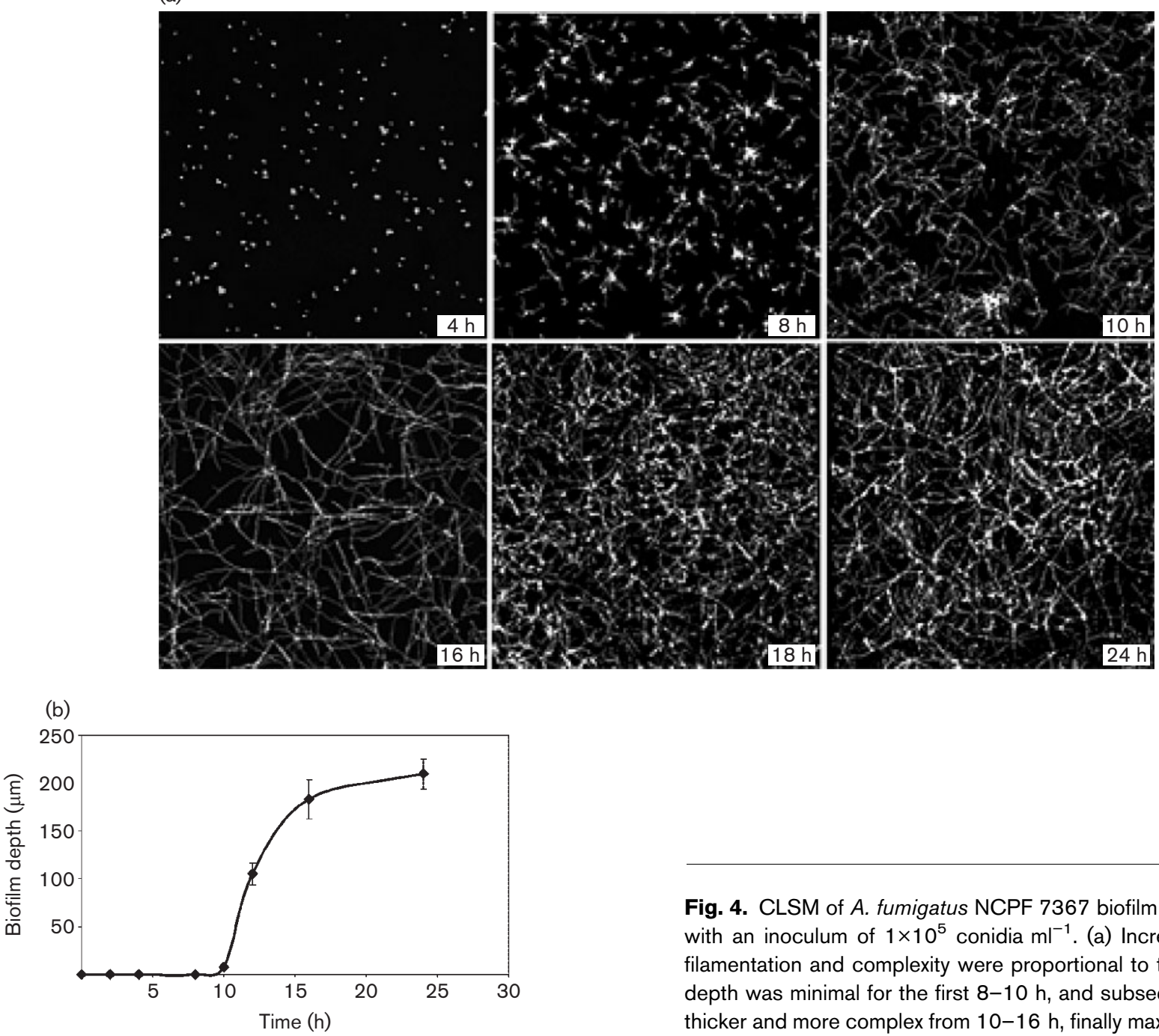

Fig. 4. CLSM of $A$. fumigatus NCPF 7367 biofilm growth kinetics with an inoculum of $1 \times 10^{5}$ conidia $\mathrm{ml}^{-1}$. (a) Increasing levels of filamentation and complexity were proportional to time. (b) Biofilm depth was minimal for the first 8-10 h, and subsequently became thicker and more complex from 10-16 h, finally maximizing at $24 \mathrm{~h}$.

various antimicrobial agents (Ramage et al., 2001a; Tunney et al., 2004). There may be limitations to this assay, such as viable but non-culturable organisms. However, it is unlikely that a high proportion of the biofilm organisms are composed of these viable but non-culturable cells. Overall, the assay enables the user to assess rapidly, either by naked eye or by spectroscopic analysis, the concentration of antifungal agent required to kill or inhibit $A$. fumigatus.

Table 1. Biofilm susceptibility testing

Concentrations are given as $\mathrm{mg}^{-1}$.

\begin{tabular}{|c|c|c|c|c|c|c|c|c|c|c|c|c|}
\hline \multirow[t]{2}{*}{ Isolate } & \multicolumn{3}{|c|}{ Itraconazole } & \multicolumn{3}{|c|}{ Voriconazole } & \multicolumn{3}{|c|}{ AmpB } & \multicolumn{3}{|c|}{ Caspofungin } \\
\hline & PMIC & SMIC $_{50}$ & $\mathrm{SMIC}_{90}$ & PMIC & $\mathrm{SMIC}_{50}$ & $\mathrm{SMIC}_{90}$ & PMIC & $\mathrm{SMIC}_{50}$ & $\mathrm{SMIC}_{90}$ & PMIC & $\mathrm{SMIC}_{50}$ & SMIC $_{90}$ \\
\hline NCPF 7367 & 0.5 & $>256$ & $>256$ & 0.25 & 16 & $>256$ & 1.0 & 0.125 & 32 & 0.25 & 128 & $>256$ \\
\hline YHCF1 & 0.5 & $>256$ & $>256$ & 0.25 & 16 & $>256$ & 1.0 & 0.125 & 16 & 0.25 & 128 & $>256$ \\
\hline YHCF2 & 0.5 & $>256$ & $>256$ & 1.0 & 128 & $>256$ & 0.25 & $<0.125$ & 8 & 0.25 & 64 & $>256$ \\
\hline YHCF3 & 0.5 & $>256$ & $>256$ & 0.5 & 32 & $>256$ & 1.0 & $<0.125$ & 32 & 0.25 & 64 & $>256$ \\
\hline YHCF4 & 0.25 & $>256$ & $>256$ & 0.25 & 16 & $>256$ & 0.5 & 1 & 16 & 0.25 & 64 & $>256$ \\
\hline
\end{tabular}


In this study, five strains of $A$. fumigatus were examined for their susceptibility to a range of antifungal agents when grown as a complex mycelial structure, using a methodology previously employed for $C$. albicans biofilms (Ramage et al., 2001a). These agents included two azoles (itraconazole and voriconazole), a polyene (AmpB) and an echinocandin (caspofungin), which were serially diluted and used to challenge planktonic and sessile cells. Table 1 illustrates the results from both conventional planktonic CLSI M38-A susceptibility testing and the modified sessile susceptibility testing. The results indicated that caspofungin had a PMIC of $0.25 \mathrm{mg} \mathrm{l}^{-1}$, itraconazole 0.25 $0.5 \mathrm{mg} \mathrm{l}^{-1}$, voriconazole $0.25-1 \mathrm{mg} \mathrm{l}^{-1}$ and $\mathrm{AmpB}$ $0.25-1 \mathrm{mg} \mathrm{l}^{-1}$. Testing of the sessile cells showed that AmpB was the most effective antifungal agent, with $\mathrm{SMIC}_{50}$ values ranging from $<0.125$ to $1 \mathrm{mg} \mathrm{l}^{-1}$ and $\mathrm{SMIC}_{90}$ values from 8 to $32 \mathrm{mg} \mathrm{l}^{-1}$. The other azole, voriconazole, exhibited increased efficacy $\left(\mathrm{SMIC}_{50}\right.$ of $16-128 \mathrm{mg} \mathrm{l}^{-1}$ ). Caspofungin showed poor overall activity against adherent multicellular A. fumigatus, with $\mathrm{SMIC}_{50}$ and $\mathrm{SMIC}_{90}$ values of 64-128 and $>256 \mathrm{mg} \mathrm{l}^{-1}$, respectively. Itraconazole was ineffective against all adherent multicellular populations $\left(\mathrm{SMIC}_{50}\right.$ and $\mathrm{SMIC}_{90}$ values of $>256 \mathrm{mg}$ ). Overall, AmpB was the most effective against sessile cells at the lowest concentrations, followed by voriconazole, caspofungin and itraconazole. It has been shown previously that azole antifungals were ineffective against C. albicans and Candida dubliniensis biofilms, whereas echinocandins were the most effective (Ramage et al., 2001a, b, c). Hawser et al. (2001) reported that $A$. fumigatus isolates were more susceptible to echinocandins using conidial concentrations equivalent to the CLSI M38-A method combined with XTT measurements. However, in this study, with an increased cell biomass, caspofungin was ineffective. The inability to successfully treat IA patients with caspofungin has been reported elsewhere, with only $41 \%$ responding during salvage therapy (Maertens et al., 2004). We note, however, that both voriconazole and $\mathrm{AmpB}$ had the ability to reduce cellular viability by over $50 \%$ at relatively low concentrations (Table 1). Therefore, although total death was not achieved, there was a certain degree of efficacy against these tenacious multicellular structures, which has been reported previously for other in vitro fungal biofilms (Ramage et al., 2002c). This may be an important observation when these agents are used empirically, as they may have a role in preventing the establishment of a mature biofilm. More work will be required to elucidate this.

How do these results relate to clinical practice? A recent review of empirical antifungal therapy in neutropenic patients compared 13 studies. The success rates of the treatments reported showed variations from 31 to $86 \%$ (Martino \& Viscoli, 2006). This variability in outcome may be as much to do with the patient population, the weakness of the indications for treatment and the consequent difficulty in establishing objective and reproducible end points for comparisons as the effectiveness of the antifungal agents. The time that treatment is started in relation to the development of the mature biofilm may also impact on the outcome. The optimal time for starting antifungal therapy in neutropenic patients remains undetermined, although most experts recommend waiting until day 5 or 7 of persistent fever (Bennett et al., 2003). It may be more prudent to start treatment before the multicellular structure has established, but this will require further in vitro studies. In CF, there is recognition that $A$. fumigatus in sputum cultures in the absence of ABPA may be a pathogen that can directly cause respiratory exacerbations. Antifungal therapy should be considered when deteriorating respiratory function is not responding to antibacterial therapy. Treatment with antifungal agents has been evaluated in these patients and an improvement in clinical condition observed (Shoseyov et al., 2006). Nevertheless, more studies are required before the effectiveness of antifungal agents in this group of patients can be evaluated fully.

Overall, this study demonstrated that A. fumigatus grows as a complex, multicellular biofilm and that the concentration of antifungal drug required for the effective treatment of these biofilm-related infections is distinct from assessment by the standard CLSI M38-A assay. This standard method of susceptibility testing does not give a true evaluation of the susceptibility of the disease-causing organism to a given antifungal agent. This may have implications for the diagnosis and management of patients with both invasive and non-invasive infections with this organism. Future studies to examine changes in gene expression of these biofilm-associated organisms may provide ways of elucidating new therapeutic options for controlling A. fumigatus biofilms in immunocompromised individuals.

\section{ACKNOWLEDGEMENTS}

We would like to thank Helen Kennedy for supplying the A. fumigatus strains from the Royal Hospital for Sick Children (Yorkhill Division, NHS Glasgow, UK). This study was supported by an educational grant from Pfizer.

\section{REFERENCES}

Antachopoulos, C., Meletiadis, J., Roilides, E., Sein, T. \& Walsh, T. J. (2006). Rapid susceptibility testing of medically important zygomycetes by XTT assay. J Clin Microbiol 44, 553-560.

Beauvais, A., Schmidt, C., Guadagnini, S., Roux, P., Perret, E., Henry, C., Paris, S., Mallet, A., Prévost, M.-C. \& Latgé, J. P. (2007). An extracellular matrix glues together the aerial-grown hyphae of Aspergillus fumigatus. Cell Microbiol 9, 1588-1600.

Bennett, J. E., Powers, J., Walsh, T., Viscoli, C., de Pauw, B., Dismukes, W., Galgiani, J., Glauser, M., Herbrecht, R. \& other authors (2003). Forum report: issues in clinical trials of empirical antifungal therapy in treating febrile neutropenic patients. Clin Infect Dis 36, S117-S122.

Brakhage, A. A. (2005). Systemic fungal infections caused by Aspergillus species: epidemiology, infection process and virulence determinants. Curr Drug Targets 6, 875-886.

Chandra, J., Kuhn, D. M., Mukherjee, P. K., Hoyer, L. L., McCormick, T. \& Ghannoum, M. A. (2001). Biofilm formation by the fungal pathogen 
Candida albicans: development, architecture, and drug resistance. J Bacteriol 183, 5385-5394.

Christensen, G. D., Simpson, W. A., Younger, J. J., Baddour, L. M., Barrett, F. F., Melton, D. M. \& Beachey, E. H. (1985). Adherence of coagulase-negative staphylococci to plastic tissue culture plates: a quantitative model for the adherence of staphylococci to medical devices. J Clin Microbiol 22, 996-1006.

Cimon, B., Symoens, F., Zouhair, R., Chabasse, D., Nolard, N., Defontaine, A. \& Bouchara, J. P. (2001). Molecular epidemiology of airway colonisation by Aspergillus fumigatus in cystic fibrosis patients. J Med Microbiol 50, 367-374.

Costerton, J. W., Lewandowski, Z., Caldwell, D. E., Korber, D. R. \& Lappin-Scott, H. M. (1995). Microbial biofilms. Annu Rev Microbiol 49, 711-745.

de Almeida, M. B., Bussamra, M. H. \& Rodrigues, J. C. (2006). Allergic bronchopulmonary aspergillosis in paediatric cystic fibrosis patients. Paediatr Respir Rev 7, 67-72.

Denning, D. W., Marinus, A., Cohen, J., Spence, D., Herbrecht, R., Pagano, L., Kibbler, C., Kcrmery, V., Offner, F. \& other authors (1998). An EORTC multicentre prospective survey of invasive aspergillosis in haematological patients: diagnosis and therapeutic outcome. EORTC Invasive Fungal Infections Cooperative Group. J Infect 37, 173-180.

Ellis, M., Richardson, M. \& de Pauw, B. (2000). Epidemiology. Hosp Med 61, 605-609.

Filler, S. G. \& Sheppard, D. C. (2006). Fungal invasion of normally non-phagocytic host cells. PLoS Pathogens 2, e129.

Hawser, S. P., Norris, H., Jessup, C. J. \& Ghannoum, M. A. (1998). Comparison of a 2,3-bis(2-methoxy-4-nitro-5-sulfophenyl)-5-[(phenylamino)carbonyl]-2H-tetrazolium hydroxide (XTT) colorimetric method with the standardized National Committee for Clinical Laboratory Standards method of testing clinical yeast isolates for susceptibility to antifungal agents. J Clin Microbiol 36, 1450-1452.

Hawser, S. P., Jessup, C., Vitullo, J. \& Ghannoum, M. A. (2001). Utility of 2,3-bis(2-methoxy-4-nitro-5-sulfophenyl)-5-[(phenyl-amino)carbonyl]-2H-tetrazolium hydroxide (XTT) and minimum effective concentration assays in the determination of antifungal susceptibility of Aspergillus fumigatus to the lipopeptide class compounds. J Clin Microbiol 39, 2738-2741.

Herbrecht, R., Denning, D. W., Patterson, T. F., Bennett, J. E., Greene, R. E., Oestmann, J. W., Kern, W. V., Marr, K. A., Ribaud, P. \& other authors (2002). Voriconazole versus amphotericin B for primary therapy of invasive aspergillosis. N Engl J Med 347, 408-415.

Hope, W. W., Walsh, T. J. \& Denning, D. W. (2005). Laboratory diagnosis of invasive aspergillosis. Lancet Infect Dis 5, 609-622.

Kamai, Y., Chiang, L. Y., Lopes Bezerra, L. M., Doedt, T., Lossinsky, A. S., Sheppard, D. C. \& Filler, S. G. (2006). Interactions of Aspergillus fumigatus with vascular endothelial cells. Med Mycol 44 (Suppl. 1), S115-S117.

Latge, J. P. (2001). The pathobiology of Aspergillus fumigatus. Trends Microbiol 9, 382-389.

Maertens, J., Raad, I., Petrikkos, G., Boogaerts, M., Selleslag, D., Petersen, F. B., Sable, C. A., Kartsonis, N. A., Ngai, A. \& other authors (2004). Efficacy and safety of caspofungin for treatment of invasive aspergillosis in patients refractory to or intolerant of conventional antifungal therapy. Clin Infect Dis 39, 1563-1571.

Martino, R. \& Viscoli, C. (2006). Empirical antifungal therapy in patients with neutropenia and persistent or recurrent fever of unknown origin. Br J Haematol 132, 138-154.

Meletiadis, J., Meis, J. F., Mouton, J. W. \& Verweij, P. E. (2001a). Analysis of growth characteristics of filamentous fungi in different nutrient media. J Clin Microbiol 39, 478-484.
Meletiadis, J., Mouton, J. W., Meis, J. F., Bouman, B. A., Donnelly, J. P. \& Verweij, P. E. (2001b). Colorimetric assay for antifungal susceptibility testing of Aspergillus species. J Clin Microbiol 39, 3402-3408.

Meletiadis, J., Mouton, J. W., Meis, J. F., Bouman, B. A., Donnelly, P. J. \& Verweij, P. E. (2001c). Comparison of spectrophotometric and visual readings of NCCLS method and evaluation of a colorimetric method based on reduction of a soluble tetrazolium salt, 2,3-bis [2methoxy-4-nitro-5-[(sulfenylamino) carbonyl]-2H-tetrazoliumhydroxide], for antifungal susceptibility testing of Aspergillus species. J Clin Microbiol 39, 4256-4263.

Mensi, M., Salgarello, S., Pinsi, G. \& Piccioni, M. (2004). Mycetoma of the maxillary sinus: endodontic and microbiological correlations. Oral Surg Oral Med Oral Pathol Oral Radiol Endod 98, 119-123.

NCCLS (2002). Reference Method for Broth Microdilution Antifungal Susceptibility Testing of Filamentous Fungi. NCCLS document M38-A. Pennsylvania, PA: National Committee for Clinical Laboratory Standards.

O'Toole, G. A. \& Kolter, R. (1998). Initiation of biofilm formation in Pseudomonas fluorescens WCS365 proceeds via multiple, convergent signalling pathways: a genetic analysis. Mol Microbiol 28, 449-461.

Ramage, G., Vande Walle, K., Wickes, B. L. \& Lopez-Ribot, J. L. (2001a). Standardized method for in vitro antifungal susceptibility testing of Candida albicans biofilms. Antimicrob Agents Chemother 45, 2475-2479.

Ramage, G., Vande Walle, K., Wickes, B. L. \& Lopez-Ribot, J. L. (2001b). Biofilm formation by Candida dubliniensis. J Clin Microbiol 39, 3234-3240.

Ramage, G., Vandewalle, K., Wickes, B. L. \& Lopez-Ribot, J. L. (2001c). Characteristics of biofilm formation by Candida albicans. Rev Iberoam Micol 18, 163-170.

Ramage, G., Bachmann, S., Patterson, T. F., Wickes, B. L. \& LopezRibot, J. L. (2002a). Investigation of multidrug efflux pumps in relation to fluconazole resistance in Candida albicans biofilms. J Antimicrob Chemother 49, 973-980.

Ramage, G., Saville, S. P., Wickes, B. L. \& Lopez-Ribot, J. L. (2002b). Inhibition of Candida albicans biofilm formation by farnesol, a quorum-sensing molecule. Appl Environ Microbiol 68, 5459-5463.

Ramage, G., VandeWalle, K., Bachmann, S. P., Wickes, B. L. \& Lopez-Ribot, J. L. (2002C). In vitro pharmacodynamic properties of three antifungal agents against preformed Candida albicans biofilms determined by time-kill studies. Antimicrob Agents Chemother 46, 3634-3636.

Ramage, G., Saville, S. P., Thomas, D. P. \& Lopez-Ribot, J. L. (2005). Candida biofilms: an update. Eukaryot Cell 4, 633-638.

Rivera, A., Hohl, T. \& Pamer, E. G. (2006). Immune responses to Aspergillus fumigatus infections. Biol Blood Marrow Transplant 12, 47-49.

Shibuya, K., Ando, T., Hasegawa, C., Wakayama, M., Hamatani, S., Hatori, T., Nagayama, T. \& Nonaka, H. (2004). Pathophysiology of pulmonary aspergillosis. J Infect Chemother 10, 138-145.

Shoseyov, D., Brownlee, K. G., Conway, S. P. \& Kerem, E. (2006). Aspergillus bronchitis in cystic fibrosis. Chest 130, 222-226.

Singh, N. (2005). Invasive aspergillosis in organ transplant recipients: new issues in epidemiologic characteristics, diagnosis, and management. Med Mycol 43 (Suppl. 1), S267-S270.

Tunney, M. M., Ramage, G., Field, T. R., Moriarty, T. F. \& Storey, D. G. (2004). Rapid colorimetric assay for antimicrobial susceptibility testing of Pseudomonas aeruginosa. Antimicrob Agents Chemother 48, 1879-1881.

Villena, G. K. \& Gutierrez-Correa, M. (2006). Production of cellulase by Aspergillus niger biofilms developed on polyester cloth. Lett Appl Microbiol 43, 262-268. 